\title{
Violence, runaway, and suicide attempts among people living with schizophrenia in China: Prevalence and correlates
}

\author{
Yixiang Long ${ }^{1}$, Xiaoliang Tong ${ }^{\text {Corresp., }}{ }^{1}$, Michael Awad ${ }^{2}$, Shijun $\mathbf{X i}^{3}, \mathbf{Y u ~ Y u}^{2,3}$ \\ ${ }^{1}$ Department of Nursing, Third Xiangya Hospital, Central South University, Changsha, Hunan, China \\ Division of Prevention and Community Research, Department of Psychiatry, Yale University School of Medicine, New Haven, Connecticut, USA \\ 3 Department of Social Medicine and Health Management, Xiangya School of Public Health, Central South University, Changsha, Hunan, China \\ Corresponding Author: Xiaoliang Tong \\ Email address: 403616846@qq.com
}

Background. People living with schizophrenia are at higher risk of disruptive behaviors, including violence, running away from home, and suicide attempts, which often co-occurs and are highly correlated, yet seldom studied together. The current study investigated the frequency and correlates of disruptive behaviors among a Chinese community sample of individuals living with schizophrenia.

Methods. A cross-sectional study was conducted among 400 individuals living with schizophrenia from 12 communities. Data about disruptive behaviors in the past 2 months was collected using self-designed questionnaires. Clinical characteristics including psychiatric symptoms, depression, anxiety, disability, and functioning were collected by internationally standardized assessment instruments.

Results. About one-fifth (21\%) of the subjects had experienced at least one form of disruptive behavior in the past 2 months. Violence was the most commonly reported (17.25\%), which included damaging property (15\%) and physical violence toward others (7.5\%); followed by running away (6.5\%), and suicide attempts (4\%). Logistic regression analysis suggested that medication non-adherence $(O R=4.96,95 \%$ $\mathrm{Cl}: 1.79-13.72)$, involuntary hospital admission ( $\mathrm{OR}=5.35,95 \% \mathrm{Cl}: 2.06-13.87)$, depression $(\mathrm{OR}=2.34,95 \%$ $\mathrm{Cl}: 1.07-5.10)$, and lower social functioning $(\mathrm{OR}=0.97,95 \% \mathrm{Cl}: 0.93-0.99)$ were independently associated with a higher risk of disruptive behaviors.

Conclusions. The overlap among three forms of disruptive behaviors warrants them to be assessed and studied together in clinical, research, and policy fields. The significant association between disruptive behaviors with medication non-adherence, involuntary admission, depression, and lower social functioning indicates the need for integrated, targeted, and needs-based intervention programs to be developed for the prevention and treatment of these disruptive behaviors. 


\section{Violence, runaway, and suicide attempts among people living with}

\section{schizophrenia in China: Prevalence and correlates}

1 Yixiang Long ${ }^{1}$, Xiaoliang Tong ${ }^{1 *}$, Michael Awad ${ }^{2}$, Shi-jun $\mathbf{X i}^{3}, \mathbf{Y u} \mathbf{Y u}^{2,3}$

$2{ }^{1}$ Department of Nursing, Third Xiangya Hospital, Central South University, Changsha, Hunan,

3 China

$4 \quad 2$ Division of Prevention and Community Research, Department of Psychiatry, Yale University

5 School of Medicine, New Haven, Connecticut, USA

$6 \quad{ }^{3}$ Department of Social Medicine and Health Management, Xiangya School of Public Health,

7 Central South University, Changsha, Hunan, China

8

$9 *$ Correspondence:

10 Xiaoliang Tong ${ }^{1}$

11 Address: 172 Tongzipo Road, Yuelu District, Changsha City, Hunan Province, 410013, China

12 Email: 601630@csu.edu.cn 


\section{Abstract}

24 Background. People living with schizophrenia are at higher risk of disruptive behaviors,

25 including violence, running away from home, and suicide attempts, which often co-occurs and

26 are highly correlated, yet seldom studied together. The current study investigated the frequency

27 and correlates of disruptive behaviors among a Chinese community sample of individuals living 28 with schizophrenia.

29 Methods. A cross-sectional study was conducted among 400 individuals living with

30 schizophrenia from 12 communities. Data about disruptive behaviors in the past 2 months was

31 collected using self-designed questionnaires. Clinical characteristics including psychiatric

32 symptoms, depression, anxiety, disability, and functioning were collected by internationally

33 standardized assessment instruments.

34 Results. About one-fifth (21\%) of the subjects had experienced at least one form of disruptive

35 behavior in the past 2 months. Violence was the most commonly reported (17.25\%), which

36 included damaging property (15\%) and physical violence toward others $(7.5 \%)$; followed by

37 running away (6.5\%), and suicide attempts (4\%). Logistic regression analysis suggested that

38 medication non-adherence $(O R=4.96,95 \% C I: 1.79-13.72)$, involuntary hospital admission

$39(O R=5.35,95 \% C I: 2.06-13.87)$, depression $(O R=2.34,95 \% C I: 1.07-5.10)$, and lower social

40 functioning $(O R=0.97,95 \% C I: 0.93-0.99)$ were independently associated with a higher risk of

41 disruptive behaviors.

42 Conclusions. The overlap among three forms of disruptive behaviors warrants them to be

43 assessed and studied together in clinical, research, and policy fields. The significant association

44 between disruptive behaviors with medication non-adherence, involuntary admission, 
45 depression, and lower social functioning indicates the need for integrated, targeted, and needs-

46 based intervention programs to be developed for the prevention and treatment of these disruptive

47 behaviors.

48 Keywords: Disruptive behaviors, Violence; Runaway; Suicide; Schizophrenia; Prevalence;

49 Correlates; China

50

51

52

53

54

55

56

57

\section{Introduction}

Schizophrenia is a debilitating, persistent psychiatric disorder characterized by symptoms such as delusions, hallucinations, and lack of emotional responsiveness and motivation, which significantly impair a person's cognition and social functioning (APA 1994). Globally, people living with schizophrenia are at a higher risk of a range of disruptive behaviors, including physical violence (Bottesi et al. 2021; Brown et al. 2019; Fazel et al. 2009; Slamanig et al. 2021), running away from home (Jaafari et al. 2019), and suicide (Ayalew et al. 2021; De Sousa et al. 2020; Lyu et al. 2021; Ran et al. 2020; Sher \& Kahn 2019), than the general population. A pooled estimated prevalence of $18.5 \%-35.4 \%$ for violence among schizophrenia individuals and other psychosis has been reported in various reviews (Large \& Nielssen 2011; Li et al. 2020; Witt et al. 2013; Zhou et al. 2016), with risk estimated at seven times that of the general population in both retrospective and cohort studies (Mullen et al. 2000; Tiihonen et al. 1997). High rates of running away from home have also been reported among people with mental illness (Jaafari et al. 2019), although less studied among individuals living with schizophrenia. For suicide, a lifetime suicide rate of 5\%-10\% has been reported among individuals living with schizophrenia by various reviews (Hor \& Taylor 2010; Sher \& Kahn 2019), with standardized mortality ratios between 10 and 20 (Hor \& Taylor 2010). 
Although violence, running away, and suicide have been viewed as distinct disruptive

69

behaviors, accumulating evidence has demonstrated that they often co-occur and are highly correlated with each other (Meltzer et al. 2012; Witt et al. 2014). However, research has focused on these behaviors separately, and rates and risk factors for any disruptive behaviors among individuals living with schizophrenia are rarely reported in the same study. Several reviews have summarized a range of risk factors for violence among individuals living with schizophrenia, including socio-demographics, past history, treatment-related factors, psychiatric symptoms, mood disorders, substance abuse, etc. (Rund 2018; Witt et al. 2013). Similar risk factors have also been reported for runaway and suicide attempts among individuals living with schizophrenia (Jaafari et al. 2019; Sher \& Kahn 2019; Shlafer et al. 2012). In light of the overlapping risk factors shared by violence, running away, and suicide, it is thus both interesting and important to take into account all three forms of disruptive behaviors simultaneously to gain a more integrated and inclusive picture of disruptive behaviors among individuals living with schizophrenia.

In China, increasing attention has been paid to disruptive behaviors of people with serious mental illness, with several national policies, regulations, and programs initiated successively by the Chinese government. In 2004, the Chinese government started the 686 Program with one important goal being identifying and treating people with serious mental illness who are at high risk of disruptive behaviors (Ma 2012). In 2011, the Chinese government launched its first National System of Basic Information Collection and Analysis for Psychoses mainly to collect data on their violent or socially-disruptive behaviors (Zhou \& Xiao 2015). In 2012, China passed its first National Mental Health Law allowing for involuntary admission of people with serious mental illness who are highly violent (Shao \& Xie 2013). In 2016, the 
90 Chinese government issued a Reward Policy to financially incentivize and reward family

91 management of disruptive behaviors of persons with serious mental illness (Yu et al. 2018).

92 Although great political attention has been directed towards disruptive behaviors of

93 serious mental illness in the community, little research has been done in this area. Most of the

94 existing research on this topic has been hospital-based (Zhou et al. 2016). Since the majority of

95 individuals with schizophrenia are living in the community, community-based research on

96 disruptive behaviors is warranted. In addition, disruptive behaviors including violence, running

97 away, and suicide have been mostly studied separately both in China and abroad, although

98 increasing evidence has shown high overlaps among these behaviors and their risk factors

99 (Jaafari et al. 2019; Sher \& Kahn 2019; Shlafer et al. 2012; Witt et al. 2014). There is a lack of

100 research simultaneously studying the three forms of disruptive behaviors in the same sample. In

101 response to these limitations, we conducted the current study to investigate the prevalence and

102 risk factors of three common forms of disruptive behaviors: violence, running away, and suicide

103 in a community sample of individuals living with schizophrenia in China.

\section{Materials \& Methods}

106 Study population

107 This cross-sectional study was conducted in Changsha Psychiatric Hospital from May

1082019 to September 2019 as a baseline assessment of a WeChat-based family intervention project

109 focused on improving the family burden of people living with schizophrenia in China (Yu et al.

110 2020a). The hospital provides community-based mental health care in Changsha City through the

111686 Program, China's largest demonstration project in mental health to provide free mental

112 health care for people with serious mental illness (Ma 2012). A two-stage cluster-sampling 
113 method was adopted to identify participants. In the first stage, we randomly selected the

114 following four districts out of the 9 administrative districts of Changsha City: Gaoxin District,

115 Furong District, Kaifu District, and Tianxin District. In the second stage, we randomly selected

$1162-4$ communities from each district based on population size and the registered number of clients

117 in the 686 program. Our final sampling frame is all 1068 registered clients with serious mental

118 illness from the 12 communities that were representative of Changsha city. The inclusion criteria

119 included: (1) registered in the 686 Program; (2) diagnosed as schizophrenia by the Chinese

120 Classification of Mental Disorders-3 (CCMD-3) or the International Classification of Diseases-

12110 (ICD-10); (3) aged 18 and older; (4) living with at least one family member; (5) able to read

122 and verbally-communicate; (6) visited the community health center for each monthly free

123 medicine refill during the study periods. The exclusion criteria included: (1) had a diagnosis

124 other than schizophrenia, (2) absent for five monthly medicine refills at the community health

125 center during the five-month study period. Based on the exclusion criteria, 618 clients were

126 excluded, leading to 450 eligible clients. Among the 450 clients approached for study

127 participation, 50 didn't complete the study due to refusal of participation or dropping out during

128 the study, leading to a final sample of 400 clients with a response rate of $88.89 \%$. Figure 1 shows

129 the flow chart of participant enrollment.

130 Each month, a team of at least 3 psychiatrists from the Changsha Psychiatric Hospital

131 circulated the 12 communities to distribute free medication and run routine check-ups. Our

132 research team followed the psychiatry team during their monthly community visits and

133 approached potential participants when they came for medication refills. The research team

134 explained the details of the study purpose and procedures and conducted face-to-face interviews

135 with the participants after obtaining written informed consent from them. In addition, the 
136 psychiatrist team assessed the symptoms and functioning of each participant using standardized

137 assessment instruments. Each interview took approximately 20-30 minutes, and each participant

138 was reimbursed with 10 Renminbi (\$1.40 in 2019 dollars) after the interview. The study was

139 reviewed and approved by the Institutional Review Board of the Xiangya School of Public

140 Health, Central South University (No.: XYGW-2019-029).

141 Instruments

142 Sample characteristics Socio-demographic information including gender, age, marital

143 status, education, and employment were collected by a self-designed personal information sheet.

144 In addition, we collected clinical characteristics such as medication non-adherence and

145 involuntary admission in the past six months. Since most of the registered clients in the 686

146 Program followed the doctor's advice and took medications on a daily basis (Yu et al. 2017), we

147 defined medication non-adherence as "not taking medicine on a daily basis each month".

148 Involuntary admission was defined as "being sent to the hospital by the family members due to

149 concerns of potential violence risk the client may have when the client herself/himself doesn't

150 agree to be hospitalized." Disruptive behaviors and other clinical characteristics were measured

151 using scales which are described as below:

152 Disruptive behaviors. Disruptive behaviors including violence, running away, and suicide

153 attempts were assessed by asking whether the participants had the following four behaviors in

154 the past two months: damaging properties (violence), physical violence (violence), suicide

155 attempts, and running away from home. Damaging properties were defined as any forms of

156 aggressive behaviors towards objects, such as breaking windows, smashing up furniture, and

157 other forms of property destruction. Physical violence was defined as any form of aggressive

158 behavior toward other people such as scratching, punching, and hitting people. Suicide attempts

Peer] reviewing PDF | (2021:11:67466:1:0:NEW 24 Jan 2022) 
159 were defined as any form of non-fatal, self-directed, potentially injurious behavior with an intent

160 to die. Running away from home was defined as escaping the family caregiver's custody and

161 leaving home without advance notice and permission from the family members, thus leading to

162 family to look for them everywhere.

163 Psychiatric symptoms. Psychiatric symptoms were assessed by the 18-item Brief

164 Psychiatric Rating Scale (BPRS-18) that includes five domains of clinical symptoms: affect,

165 positive symptoms, negative symptoms, resistance, and activation (Shafer 2005). The total score

166 ranges from $0-126$, with a higher score representing more severe psychiatric symptoms. The

167 BPRS-18 showed good internal consistency with a Cronbach's alpha of 0.85 in the current study.

168 Depression. Depression was assessed by the 9-item Patient Health Questionnaire-9

169 (PHQ-9) for screening of depressive symptoms in the past two weeks (Spitzer et al. 1999). The

170 total score ranges from 0 to 27 , with a higher score indicating more depressive symptoms, and a

171 cut-off point of 10 differentiating depression and non-depression (Manea et al. 2015). The PHQ-

1729 showed good internal consistency with a Cronbach's alpha of .92 in the current study.

173 Anxiety. Anxiety was assessed by the 7-item Generalized Anxiety Disorder Scale-7

174 (GAD-7) for screening of anxiety symptoms in the past two weeks (Schalet et al. 2014). The

175 total score ranges from 0 to 21 , with a higher score indicating more anxiety symptoms, and a cut-

176 off point of 10 differentiating anxiety and non-anxiety (Schalet et al. 2014). The GAD-7 showed

177 good internal consistency with a Cronbach's alpha of .96 in the current study.

178 Disability. Disability was assessed by the 12-item World Health Organization Disability

179 Assessment Schedule 2.0 (WHODAS 2.0) for disability and functional impairment. The total

180 score ranges from $0-48$, with a higher score representing a higher level of disability. The

181 WHODAS 2.0 showed good internal consistency (Cronbach's alpha of 0.89) in the current study. 
183 for psychological, social, and occupational functioning. It is a one-item scale measured on a

184 hypothetical continuum of functionality ranging from 1 to 100 , with a higher score indicating

185 better patient functioning (Goldman et al. 1992).

186 Statistical analysis

187 Exploratory and summary statistics were obtained for all variables within the dataset.

188 Socio-demographic and clinical characteristics were compared between the disruptive group and

189 non-disruptive group by $\chi^{2}$ test for categorical variables or $t$-test for continuous variables. A

190 multivariate logistic regression was further conducted to determine correlates of disruptive

191 behaviors. All data were analyzed using STATA version 16.

\section{Results}

\section{Prevalence of disruptive behaviors}

194 Table 1 shows the prevalence of each disruptive behavior committed by the participants.

195 Approximately one fifth (21.0\%) had engaged in at least one form of disruptive behavior in the

196 past two months, with damaging properties being the most commonly reported (15.0\%),

197 followed by physical violence toward other people (7.5\%), running away from home (6.5\%), and 198 suicide attempts (4.0\%). The prevalence of violence was $17.25 \%$ when combining damaging

199 properties and attacking people. Among the disruptive group, most had only engaged in one

200 disruptive behavior (61.9\%), while less than one-third (26.2\%) had engaged in two concurrent

201 disruptive behaviors. A further comparison of disruptive behaviors by gender showed non-

202 significant differences.

\section{Comparison between disruptive and non-disruptive group}


Table 2 shows the socio-demographic and clinical characteristics of the participants and

205 their comparisons between the disruptive and non-disruptive groups. In general, the sample corresponds to a profile of middle-aged (mean age: $46.87 \pm 10.99)$, married (43.00\%), and unemployed $(89.50 \%)$ man/woman $(50 \%$ each), with middle and high school education non-disruptive groups demonstrated no significant differences except for employment, where the disruptive group had a higher unemployment rate than the non-disruptive group $(96.43 \%$ vs $87.66 \%, p=0.020)$.

With regards to clinical characteristics, only $7.20 \%$ had medication non-adherence, and relatively common among the participants, with $40.51 \%$ and $29.74 \%$ screened positive for depression and anxiety, respectively. The total sample had a mean score of $32.90 \pm 11.43$ for psychiatric symptoms, $26.02 \pm 10.22$ for disability, and $61.83 \pm 13.58$ for functioning. A further comparison of clinical characteristics between disruptive and non-disruptive groups showed significant differences in each of the measured characteristics. Compared to the non-disruptive 219 group, the disruptive group had higher rates of medication non-adherence $(15.48 \%$ vs $4.92 \%$, $p=0.001)$, involuntary admission $(20.24 \%$ vs $5.06 \%, p<0.01)$, depression $(67.07 \%$ vs $33.44 \%$, $p<0.01)$ and anxiety $(53.01 \%$ vs $23.45 \%, p<0.01)$. The disruptive group also showed more severe

$223(30.54 \pm 10.67$ vs $24.77 \pm 9.74, p<0.01)$, and lower levels of functioning $(53.03 \pm 14.05$ vs

$22464.21 \pm 12.45, p<0.01)$ than the non-disruptive group.

\section{Correlates of disruptive behaviors}


Table 3 shows a multivariate logistic regression for correlates of disruptive behaviors,

227 with disruptive behavior as the dependent variable and all socio-demographic and clinical

228 characteristics as the independent variables. Among the 12 factors that were included in the

229 model, four factors remained significant after controlling for all the other factors: medication

230 non-adherence, involuntary admissions, depression, and functioning. The risk of disruptive

231 behavior increased by four times among those with medication non-adherence $(O R: 4.96,95 \%$

232 CI: 1.79-13.72), and involuntary admission (OR:5.35, 95\% CI: 2.06-13.87) than their

233 counterparts. Compared to those without depression, those with depression were over twice as

234 likely to engage in disruptive behaviors (OR:2.34,95\% CI: 1.07-5.10). On the other hand, every

235 one-point increase in functioning decreased the risk of disruptive behavior by $3 \%(O R: 0.97,95 \%$

236 CI: 0.93-0.99). No significant association was found between disruptive behavior with age,

237 gender, marriage, education, employment, psychiatric symptoms, anxiety, or disability.

\section{Discussion}

To our knowledge, this is the first study to simultaneously examine three forms of

240 disruptive behavior ---violence, running away from home, and suicide attempts together using a

241 representative community sample of individuals living with schizophrenia. The study showed

$24221 \%$ of individuals living with schizophrenia engaged in disruptive behaviors in the past two

243 months, including $17.25 \%$ for violence (either damaging property or physical violence toward

244 others), $6.5 \%$ for running away, and $4.0 \%$ for suicide attempts. The prevalence of violence in the

245 current study was comparable to the pooled prevalence of $18.5 \%$ reported by a review that

246 included 45,533 individuals from 110 studies (Witt et al. 2013). However, this violence rate was

247 much lower than another review reporting a 34.5\% rate of violence among first-episode

248 psychosis. This discrepancy may be explained by the relatively long duration of illness (mean 
249 duration of 20 years) in the current sample as compared to the review samples who were

250 experiencing their first episodes. This finding lends further support to the literature showing a

251 greater risk of future psychosis among individuals experiencing first-episode psychosis than

252 those in subsequent episodes (Large \& Nielssen 2011), indicating the need for early diagnosis

253 and timely treatment for schizophrenia. The $17.25 \%$ prevalence rate for violence in the current

254 study was also much lower than another meta-analysis focused on aggressive behaviors among

255 individuals living with schizophrenia in China, which reported a pooled prevalence of $35.4 \%$

256 (95\% CI: $29.7 \%, 41.4 \%$ ) (Zhou et al. 2016). This disparity may be best explained by the sample

257 differences (community samples vs. hospital samples), implying an increased risk of violence

258 among individuals living with schizophrenia in a highly restrictive and controlled environment

259 (Zhou et al. 2016).

260 Running away from home has been seldom studied among individuals living with

261 schizophrenia in Western countries. This may be because many of them are adults who live alone

262 instead of with their families. However, in Asian countries such as China, most individuals with

263 schizophrenia live with their families, which is reflected in Confucian cultural values that the

264 family should take the responsibility to live with and care for any sick member until they recover

265 (Yu et al. 2020b). Having a family member with schizophrenia who runs away from home not

266 only puts that individual at great risk of getting injured, engaging in unhealthy activities,

267 becoming homeless, and even premature death (Meltzer et al. 2012), but also brings shame on

268 their family members due to their lack of supervision and inadequate care of their loved one (Yu

269 et al. 2020b). Our study, for the first time, found a $6.5 \%$ rate of running away from home among

270 individuals living with schizophrenia, which warrants more scholarly attention and intervention

271 in this area. 
273 people with schizophrenia. An earlier review by Miles (Miles 1977) showed a lifetime attempt

274 rate of $10 \%$ for suicide associated with schizophrenia, which used to be the most cited figure by

275 past studies. However, recent studies suggest $5 \%$ as a more representative suicide attempt rate

276 associated with schizophrenia (Hor \& Taylor 2010). The 4.0\% prevalence rate of suicide

277 attempts in the current study also seems to support a lower suicide attempt, though this is still

278 significantly higher than the reported $0.9 \%$ of the general population (Hor \& Taylor 2010). One

279 implication of the current study is that risk for suicide should be included in the routine

280 assessment of individuals living with schizophrenia, with more support and resources provided

281 to those at higher risk.

282 The finding that medication non-adherence was associated with a 4-fold increased risk of

283 disruptive behavior was consistent with the bulk of studies. Non-adherence to medication among

284 individuals living with schizophrenia has been well-established to be associated with a range of

285 adverse outcomes, including violence, victimization, running away, and suicide (Appelbaum

286 2019; Witt et al. 2013). It also greatly increased the severity of these disruptive behaviors and is

287 associated with increased premature death (Appelbaum 2019; Witt et al. 2013). The mechanism

288 underlying the positive association between medication non-adherence and disruptive behaviors

289 may be explained by the exacerbation of psychiatric symptoms (especially positive symptoms)

290 after discontinuation of medication, which, in turn, leads to a series of more disruptive behaviors

291 such as violence and suicide (Appelbaum 2019; Witt et al. 2013). This finding underscores the

292 need for early intervention and sustained engagement in treatment (Buckley 2012).

293 Consistent with two reviews and meta-analyses showing a four-fold increase in violence

294 risk associated with involuntary treatment (Large \& Nielssen 2011; Witt et al. 2013), the current 
295 study found an even higher risk ratio of 5.35. Considering the highly-restrictive and controlled

296 environment and usually long length of stay associated with hospitals, it is not surprising that

297 individuals with histories of involuntary admission may have had more negative interpersonal

298 experiences prior to or during these hospitalizations that may make them more likely to engage

299 in disruptive behavior as a way of coping (Zhou et al. 2016). On the other hand, engaging in

300 disruptive behaviors may have led to involuntary admission in the first place (Large \& Nielssen

301 2011). For instance, the first Chinese Mental Health Law stipulated that people with serious

302 mental illness could be involuntarily hospitalized if they posed a risk to themselves or others

303 (Shao \& Xie 2013). This finding represents an important implication for future studies to

304 examine the dynamics between involuntary admission and disruptive behaviors. It also highlights

305 the need to develop more community-based rehabilitation programs, complementary to

306 involuntary hospitalization, in order to reduce future risk of disruptive behaviors and promote

307 recovery among individuals living with schizophrenia in the community.

308 While robust evidence has consistently shown depression to be a strong predictor for both

309 running away from home and attempting/completing suicide (Tucker et al. 2011), research on

310 depression and violence have yielded divergent and conflicting conclusions, with some research

311 showing negative associations (Ekinci \& Ekinci 2013) and some showing positive and even non-

312 significant association (Witt et al. 2013). Our findings support a positive association between

313 depression and disruptive behaviors and indicate the need to treat depression in individuals living

314 with schizophrenia. It is important to note that depressive symptoms have often been

315 misunderstood as negative symptoms of schizophrenia and thus left undiagnosed and untreated

316 among individuals with schizophrenia (Xi et al. 2021). Identifying depression and distinguishing 
317 depression from other schizophrenia symptoms should be the first key step towards decreasing

318 depression and its related disruptive behaviors in individuals with schizophrenia.

319 Despite a large body of research reporting psychiatric symptoms as a major risk factor for

320 disruptive behaviors in schizophrenia (Rund 2018; Witt et al. 2013), our current study failed to

321 show such an association in multivariate regression. Instead, we found better functioning was

322 significantly associated with decreased risk of disruptive behaviors, which has rarely been

323 reported in previous literature. One explanation may be that psychiatric symptoms and

324 functioning are two overlapping constructs that are closely related to each other, the association

325 between psychiatric symptoms and disruptive behaviors may be mediated by functioning.

326 Further research is needed to explore such association and its underlying mechanism. This

327 finding suggests improvement of functioning in schizophrenia may potentially serve an

328 important purpose in the prevention of disruptive behaviors.

329 Several limitations should be acknowledged in the interpretation of the study findings.

330 First, the sample was recruited from 12 communities in Changsha city of Hunan Province and

331 may not represent people living with schizophrenia in other parts of China. Future national level

332 and multi-center studies may be needed to provide a more comprehensive picture of disruptive

333 behaviors among people living with schizophrenia in China. Second, the cross-sectional design

334 of the study precludes any causal relationships between disruptive behaviors and risk factors,

335 future longitudinal study designs are needed to establish causal relationships. Third, the

336 prevalence of disruptive behaviors was only measured for a short time - the past two months -

337 due to the concern of recall bias, which may be lower than the most commonly used time frame

338 of six months or a lifetime. Future studies may consider using a longer time for disruptive

339 behaviors assessment. Fourth, confirmation of disruptive behaviors relies solely on an 
340 individual's self-report, which may have been underreported by participants due to social

341 desirability bias. Future studies may benefit from adding more objective assessment indicators

342 such as an informant's description, medical and judicial records. Fifth, we didn't distinguish

343 various types of schizophrenia diagnosis and thus were unable to compare disruptive behaviors

344 by specific schizophrenia subtypes. Future studies may consider studying each specific type of

345 schizophrenia and comparing the prevalence and risk factors of disruptive behaviors by

346 schizophrenia diagnosis subtypes.

347 In spite of the above-mentioned limitations, our study still has some important

348 innovations that may contribute to the literature. First, we studied three common forms of

349 disruptive behaviors simultaneously in the same study, filling in the research gap of past studies

350 that focused on only one form of disruptive behavior. Second, our sample was recruited from 12

351 communities in Changsha City and was representative of all community residents living with

352 schizophrenia in Changsha City, which was also an understudied population compared to the

353 most studied hospitalized population. Third, our results have identified four important and also

354 modifiable risk factors for disruptive behaviors and provide useful information and guidance to

355 inform future intervention and policies.

\section{Conclusion}

Disruptive behaviors including violence, running away from home, and suicide are common among people living with schizophrenia in China, which calls for early recognition and intervention of these behaviors. The risk of disruptive behaviors was higher among those with medication non-adherence, involuntary hospital admission, depression, and lower social

361 functioning, which provides implications for future targeted intervention programs to decrease

362 the risk factors so as to mitigate disruptive behaviors. 
364 Acknowledgements

365 The authors would like to thank individuals for their participation in this study, family members

366 who supported their participation, and staff from the Changsha Psychiatric Hospital and the 12

367 community health centers for their collaboration and support.

\section{References}

370

371

372

373

374

375

376

377

378

379

380

381

382

383

384

385

386

387

388

389

390

391

392

393

394

395

396

397

398

399

400

401

WHO, WHO Disability Assessment Schedule 2.0 (WHODAS2.0), 2017, http://www.who.int/classifications/icf/whodasii/en/.

APA APA. 1994. Diagnostic and Statistical Manual of Mental Disorders, 4th edn Washington, DC:American Psychiatric Association.

Appelbaum PS. 2019. In Search of a New Paradigm for Research on Violence and Schizophrenia. Am J Psychiatry 176:677-679.

Ayalew M, Defar S, and Reta Y. 2021. Suicide Behavior and Its Predictors in Patients with Schizophrenia in Ethiopia. Schizophr Res Treatment 2021:6662765.

Bottesi G, Candini V, Ghisi M, Bava M, Bianconi G, Bulgari V, Carra G, Cavalera C, Conte G, Cricelli M, Ferla MT, lozzino L, Macis A, Stefana A, de Girolamo G, and Group V-. 2021. Personality, Schizophrenia, and Violence: A Longitudinal Study: The Second Wave of the VIORMED Project. J Pers Disord 35:236-254.

Brown S, O'Rourke S, and Schwannauer M. 2019. Risk factors for inpatient violence and self-harm in forensic psychiatry: the role of head injury, schizophrenia and substance misuse. Brain Inj 33:313-321.

Buckley PF. 2012. Evidence suggests complex links between violence and schizophrenia. Interview by Bridget M. Kuehn. JAMA 308:658-659.

De Sousa A, Shah B, and Shrivastava A. 2020. Suicide and Schizophrenia: an Interplay of Factors. Curr Psychiatry Rep 22:65.

Ekinci O, and Ekinci A. 2013. Association between insight, cognitive insight, positive symptoms and violence in patients with schizophrenia. Nord J Psychiatry 67:116-123.

Fazel S, Gulati G, Linsell L, Geddes JR, and Grann M. 2009. Schizophrenia and violence: systematic review and meta-analysis. PLoS Med 6:e1000120.

Goldman HH, Skodol AE, and Lave TR. 1992. Revising axis V for DSM-IV: a review of measures of social functioning. Am J Psychiatry 149:1148-1156.

Hor K, and Taylor M. 2010. Suicide and schizophrenia: a systematic review of rates and risk factors. $J$ Psychopharmacol 24:81-90.

Jaafari M, Tabril T, Ouraghene A, Aarab C, Rammouz I, and Aalouane R. 2019. [Behavior disorders in the childhood and adolescence of schizophrenic patients: A retrospective study]. Encephale 45:221225.

Large MM, and Nielssen O. 2011. Violence in first-episode psychosis: a systematic review and metaanalysis. Schizophr Res 125:209-220.

Peer) reviewing PDF | (2021:11:67466:1:0:NEW 24 Jan 2022) 
402

403

404

405

406

407

408

409

410

411

412

413

414

415

416

417

418

419

420

421

422

423

424

425

426

427

428

429

430

431

432

433

434

435

436

437

438

439

440

441

442

443

444

445

446

447

448

Li W, Yang Y, Hong L, An FR, Ungvari GS, Ng CH, and Xiang YT. 2020. Prevalence of aggression in patients with schizophrenia: A systematic review and meta-analysis of observational studies. Asian J Psychiatr 47:101846.

Lyu J, Zhang J, and Hennessy DA. 2021. Characteristics and Risk Factors for Suicide in People with Schizophrenia in Comparison to Those without Schizophrenia. Psychiatry Res 304:114166.

Ma H. 2012. Integration of hospital and community services-the '686 Project'-is a crucial component in the reform of China's mental health services. Shanghai Arch Psychiatry 24:172-174.

Manea L, Gilbody S, and McMillan D. 2015. A diagnostic meta-analysis of the Patient Health Questionnaire-9 (PHQ-9) algorithm scoring method as a screen for depression. Gen Hosp Psychiatry 37:67-75.

Meltzer H, Ford T, Bebbington P, and Vostanis P. 2012. Children who run away from home: risks for suicidal behavior and substance misuse. J Adolesc Health 51:415-421.

Miles CP. 1977. Conditions predisposing to suicide: a review. J Nerv Ment Dis 164:231-246.

Mullen PE, Burgess P, Wallace C, Palmer S, and Ruschena D. 2000. Community care and criminal offending in schizophrenia. Lancet 355:614-617.

Ran MS, Xiao Y, Fazel S, Lee Y, Luo W, Hu SH, Yang X, Liu B, Brink M, Chan SKW, Chen EY, and Chan CL. 2020. Mortality and suicide in schizophrenia: 21-year follow-up in rural China. BJPsych Open 6:e121.

Rund BR. 2018. A review of factors associated with severe violence in schizophrenia. Nord J Psychiatry 72:561-571.

Schalet BD, Cook KF, Choi SW, and Cella D. 2014. Establishing a common metric for self-reported anxiety: linking the MASQ, PANAS, and GAD-7 to PROMIS Anxiety. J Anxiety Disord 28:88-96.

Shafer A. 2005. Meta-analysis of the brief psychiatric rating scale factor structure. Psychol Assess 17:324-335.

Shao Y, and Xie B. 2013. Operationalizing the involuntary treatment regulations of China's new mental health law. Shanghai Arch Psychiatry 25:384-386.

Sher L, and Kahn RS. 2019. Suicide in Schizophrenia: An Educational Overview. Medicina (Kaunas) 55.

Shlafer RJ, Poehlmann J, and Donelan-McCall N. 2012. Maternal jail time, conviction, and arrest as predictors of children's 15-year antisocial outcomes in the context of a nurse home visiting program. J Clin Child Adolesc Psychol 41:38-52.

Slamanig R, Reisegger A, Winkler H, de Girolamo G, Carra G, Crocamo C, Fangerau H, Markiewicz I, Heitzman J, Salize HJ, Picchioni M, and Wancata J. 2021. A Systematic Review of Nonpharmacological Strategies to Reduce the Risk of Violence in Patients With Schizophrenia Spectrum Disorders in Forensic Settings. Front Psychiatry 12:618860.

Spitzer RL, Kroenke K, and Williams JB. 1999. Validation and utility of a self-report version of PRIME-MD: the PHQ primary care study. Primary Care Evaluation of Mental Disorders. Patient Health Questionnaire. JAMA 282:1737-1744.

Tiihonen J, Isohanni M, Rasanen P, Koiranen M, and Moring J. 1997. Specific major mental disorders and criminality: a 26-year prospective study of the 1966 northern Finland birth cohort. Am J Psychiatry 154:840-845.

Tucker JS, Edelen MO, Ellickson PL, and Klein DJ. 2011. Running away from home: a longitudinal study of adolescent risk factors and young adult outcomes. J Youth Adolesc 40:507-518.

Witt K, Hawton K, and Fazel S. 2014. The relationship between suicide and violence in schizophrenia: analysis of the Clinical Antipsychotic Trials of Intervention Effectiveness (CATIE) dataset. Schizophr Res 154:61-67.

Witt K, van Dorn R, and Fazel S. 2013. Risk factors for violence in psychosis: systematic review and metaregression analysis of 110 studies. PLoS One 8:e55942. 
449

450

451

452

453

454

455

456

457

458

459

460

461

462

463

464

465

466

467
Xi SJ, Shen MX, Wang Y, Zhou W, Xiao SY, Tebes JK, and Yu Y. 2021. Depressive symptoms, anxiety symptoms, and their co-occurrence among people living with schizophrenia in China: Prevalence and correlates. J Clin Psychol.

Yu Y, Li T, Xi S, Li Y, Xiao X, Yang M, Ge X, Xiao S, and Tebes J. 2020a. Assessing a WeChat-Based Integrative Family Intervention (WIFI) for Schizophrenia: Protocol for a Stepped-Wedge Cluster Randomized Trial. JMIR Res Protoc 9:e18538.

Yu Y, Li TX, Li YL, Qiu D, Xi SJ, Xiao SY, and Tebes JK. 2020b. A cross-sectional study on spouse and parent differences in caregiving experiences of people living with schizophrenia in rural China. $B M C$ Psychiatry 20:226.

Yu Y, Liu ZW, Tang BW, Zhao M, Liu XG, and Xiao SY. 2017. Reported family burden of schizophrenia patients in rural China. PLoS One 12:e0179425.

Yu Y, Zhou W, and Xiao S. 2018. China's Reward Policy for Family Care of Persons With Serious Mental Illness. Psychiatr Serv:appips201800114.

Zhou JS, Zhong BL, Xiang YT, Chen Q, Cao XL, Correll CU, Ungvari GS, Chiu HF, Lai KY, and Wang XP. 2016. Prevalence of aggression in hospitalized patients with schizophrenia in China: A meta-analysis. Asia Pac Psychiatry 8:60-69.

Zhou W, and Xiao S. 2015. Reporting on China's mental health surveillance. Am J Psychiatry 172:314315. 
Figure 1

Flowchart of subject enrollment 
Figure 1. Flowchart of subject enrollment ${ }^{*}$

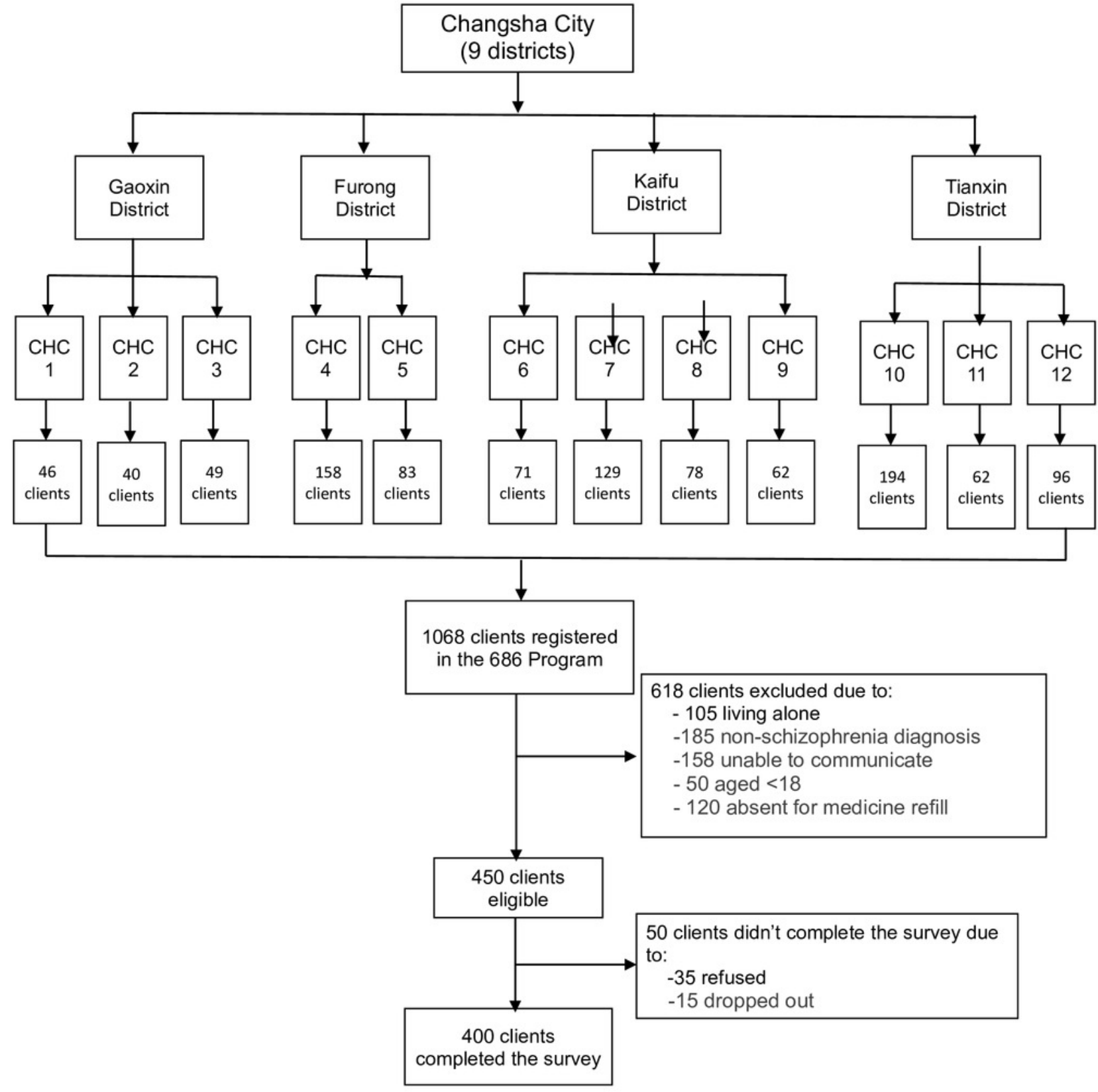

${ }^{\star} \mathrm{CHC}$ : Community health center, full name of the $12 \mathrm{CHCs}$ are listed as below:

CHC1: Gaoxin District Dongfanghong Town Health Center; CHC2: Gaoxin District Leifeng Street Community Health Service Center; CHC3: Gaoxin District Liao Jiaping Nursing Home; CHC4. Furong District Parity Hospital; CHC5. Furong District Red Cross Hospital; CHC6. Kaifu District Laodaohe Union Hospital; CHC7. Kaifu District Geriatric Hospital; CHC8. Kaifu District Hongshan Street Community Health Service Center; CHC9. Kaifu District Shaping Street Community Health Service Center; CHC10. Tianxin District Jinpenling Community Health Service Center; CHC11. Tianxin District Datuo Town Health Center; $\mathrm{CHC} 12$. Tianxin District Pozi Street Community Health Service Center 


\section{Table 1 (on next page)}

Prevalence of disruptive behaviors among schizophrenia individuals 
1

2 Table 1. Prevalence of disruptive behaviors among schizophrenia individuals $(\mathrm{n}=400)$

\begin{tabular}{|c|c|c|c|c|c|c|c|}
\hline \multirow{2}{*}{ Variables } & \multicolumn{2}{|c|}{ Total $(n=400)$} & \multicolumn{2}{|c|}{$\operatorname{Male}(n=200)$} & \multicolumn{2}{|c|}{ Female $(n=200)$} & \multirow{2}{*}{$\mathrm{P}$} \\
\hline & $\mathrm{N}$ & $\%$ & $\mathrm{~N}$ & $\%$ & $\mathrm{~N}$ & $\%$ & \\
\hline Violence & 69 & 17.25 & 35 & 17.5 & 34 & 17.0 & 0.895 \\
\hline Damaging property & 60 & 15.0 & 28 & 14.0 & 32 & 16.0 & 0.575 \\
\hline Attacking people & 30 & 7.5 & 17 & 8.5 & 13 & 6.5 & 0.448 \\
\hline Run away & 26 & 6.5 & 14 & 7.0 & 12 & 6.0 & 0.685 \\
\hline Suicide attempt & 16 & 4.0 & 9 & 4.5 & 7 & 3.5 & 0.610 \\
\hline Any disruptive behavior & 84 & 21.0 & 42 & 21.0 & 42 & 21.0 & 0.644 \\
\hline Only 1 form of behavior & 64 & 76.2 & 31 & 73.8 & 33 & 78.6 & 0.490 \\
\hline 2 forms of behavior & 13 & 15.5 & 6 & 14.3 & 7 & 16.7 & \\
\hline All 3 forms of behavior & 7 & 8.3 & 5 & 11.9 & 2 & 4.7 & \\
\hline
\end{tabular}

3

4 
Table 2 (on next page)

Socio-demographic and clinical characteristics by disruptive behaviors 
1 Table 2. Socio demographic and clinical characteristics by disruptive behaviors

\begin{tabular}{|c|c|c|c|c|c|c|c|}
\hline \multirow{2}{*}{$\begin{array}{c}\text { Socio- } \\
\text { demographics }\end{array}$} & \multicolumn{2}{|c|}{ Total $(n=400)$} & \multicolumn{2}{|c|}{$\begin{array}{l}\text { Non-disruptive group } \\
(\mathrm{n}=316)\end{array}$} & \multicolumn{2}{|c|}{$\begin{array}{l}\text { Disruptive group } \\
(\mathrm{n}=\mathbf{8 4})\end{array}$} & \multirow[t]{2}{*}{$\boldsymbol{P}$} \\
\hline & Mean/N & $\mathrm{SD} / \%$ & Mean/N & $\mathrm{SD} / \%$ & Mean/N & $\mathrm{SD} / \%$ & \\
\hline Age & 46.87 & 10.99 & 46.81 & 10.64 & 47.07 & 12.27 & 0.847 \\
\hline \multicolumn{8}{|l|}{ Gender } \\
\hline Male & 200 & 50 & 158 & 50.00 & 42 & 50 & 0.644 \\
\hline Female & 200 & 50 & 158 & 50.00 & 42 & 50 & \\
\hline \multicolumn{8}{|l|}{ Marriage } \\
\hline \multirow[t]{2}{*}{ Single } & 150 & 37.50 & 114 & 36.08 & 36 & 42.86 & 0.522 \\
\hline & 172 & 43.00 & 139 & 43.99 & 33 & 39.29 & \\
\hline \multicolumn{8}{|l|}{ Married/cohabited } \\
\hline Else $\mathrm{a}^{\mathrm{a}}$ & 78 & 19.50 & 63 & 19.94 & 15 & 17.86 & \\
\hline \multicolumn{8}{|l|}{ Education } \\
\hline Primary \& below & 75 & 18.75 & 54 & 17.09 & 21 & 25 & 0.221 \\
\hline Middle \& high & 271 & 67.75 & 217 & 68.67 & 54 & 64.29 & \\
\hline College \& above & 54 & 13.50 & 45 & 14.24 & 9 & 10.71 & \\
\hline \multicolumn{8}{|l|}{ Employment } \\
\hline Unemployed & 358 & 89.50 & 277 & 87.66 & 81 & 96.43 & 0.020 \\
\hline Employed & 42 & 10.50 & 39 & 12.34 & 3 & 3.57 & \\
\hline \multicolumn{8}{|c|}{ Clinical characteristics } \\
\hline \multicolumn{8}{|c|}{ Medication non-adherence } \\
\hline No & 361 & 92.80 & 290 & 95.08 & 71 & 84.52 & 0.001 \\
\hline Yes & 28 & 7.20 & 15 & 4.92 & 13 & 15.48 & \\
\hline \multicolumn{8}{|l|}{$\begin{array}{l}\text { Involuntary } \\
\text { admission }\end{array}$} \\
\hline No & 367 & 91.75 & 300 & 94.94 & 67 & 79.76 & $<0.001$ \\
\hline Yes & 33 & 8.25 & 16 & 5.06 & 17 & 20.24 & \\
\hline Symptoms (BPRS) & 32.9 & 11.43 & 30.99 & 9.97 & 40.12 & 13.58 & $<0.001$ \\
\hline \multicolumn{8}{|l|}{ Depression (PHQ-9) } \\
\hline No & 232 & 59.49 & 205 & 66.56 & 27 & 32.93 & $<0.001$ \\
\hline Yes & 158 & 40.51 & 103 & 33.44 & 55 & 67.07 & \\
\hline \multicolumn{8}{|l|}{ Anxiety (GAD-7) } \\
\hline No & 274 & 70.26 & 235 & 76.55 & 39 & 46.99 & $<0.001$ \\
\hline Yes & 116 & 29.74 & 72 & 23.45 & 44 & 53.01 & \\
\hline $\begin{array}{l}\text { Disability } \\
\text { (WHODAS) }\end{array}$ & 26.02 & 10.22 & 24.77 & 9.74 & 30.54 & 10.67 & $<0.001$ \\
\hline Functioning (GAF) & 61.83 & 13.58 & 64.21 & 12.45 & 53.03 & 14.05 & $<0.001$ \\
\hline
\end{tabular}

Note: BPRS: Brief Psychiatric Rating Scale; PHQ-9: Patient Health Questionnaire-9; GAD-7: Generalized Anxiety

3 Disorder Scale-7; WHODAS: World Health Organization Disability Assessment Schedule; GAF: Global

4 Assessment of Functioning

5 a: else includes separated, divorced, and widowed. 


\section{Table 3(on next page)}

Multivariate logistic regression for correlates of disruptive behaviors 
1 Table 3. Multivariate logistic regression for correlates of disruptive behaviors

2

\begin{tabular}{|c|c|c|c|c|}
\hline Variables & & OR & $95 \% \mathrm{CI}$ & $\mathbf{P}$ \\
\hline \multicolumn{5}{|c|}{ Socio-demographic characteristics } \\
\hline Age & & 1.01 & $0.98-1.04$ & 0.554 \\
\hline \multirow[t]{2}{*}{ Gender } & Male & ref & & \\
\hline & Female & 1.02 & $0.53-1.94$ & 0.958 \\
\hline \multirow[t]{3}{*}{ Marriage } & Single & ref & & \\
\hline & Married/cohabited & 0.91 & $0.42-1.96$ & 0.811 \\
\hline & Else $^{a}$ & 0.68 & $0.26-1.76$ & 0.423 \\
\hline \multirow[t]{3}{*}{ Education } & Primary \& below & ref & & \\
\hline & Middle \& high & 1.06 & $0.48-2.34$ & 0.877 \\
\hline & College \& above & 1.41 & $0.44-4.58$ & 0.565 \\
\hline \multirow[t]{2}{*}{ Employment } & Unemployed & ref & & \\
\hline & Employed & 0.74 & $0.20-2.77$ & 0.651 \\
\hline \multicolumn{5}{|l|}{ Clinical characteristics } \\
\hline \multirow[t]{2}{*}{ Medication non-adherence } & No & ref & & \\
\hline & Yes & 4.96 & $1.79-13.72$ & 0.002 \\
\hline \multirow[t]{2}{*}{ Involuntary admission } & No & ref & & \\
\hline & Yes & 5.35 & $2.06-13.87$ & 0.001 \\
\hline Symptoms (BPRS) & & 1.02 & $0.99-1.05$ & 0.252 \\
\hline \multirow[t]{2}{*}{ Depression (PHQ-9) } & No & ref & & \\
\hline & Yes & 2.34 & $1.07-5.10$ & 0.032 \\
\hline \multirow[t]{2}{*}{ Anxiety (GAD-7) } & No & ref & & \\
\hline & Yes & 1.34 & $0.61-2.95$ & 0.466 \\
\hline \multicolumn{5}{|l|}{ Disability (WHODAS) } \\
\hline Functioning (GAF) & & 0.97 & $0.93-0.99$ & 0.006 \\
\hline
\end{tabular}

3 Note: BPRS: Brief Psychiatric Rating Scale; PHQ-9: Patient Health Questionnaire-9; GAD-7: Generalized Anxiety

4 Disorder Scale-7; WHODAS: World Health Organization Disability Assessment Schedule; GAF: Global

5 Assessment of Functioning

6 a: else includes separated, divorced, and widowed. 